\title{
Elementos teóricos sobre riqueza y vivienda desde la perspectiva de la economía pública
}

Óscar Arcos Palma*

NORMAND Asuad**

\section{Introducción}

bordar los temas riqueza y vivienda desde el enfoque de la econo-
mía pública, remite al interés por saber cuál ha sido y cuál debería ser la función del Estado en la acumulación de la riqueza y su distribución y, desde la perspectiva de esta última, cómo se ha expresado el acceso a la vivienda por parte de la población, entendida esta, en algún sentido, como una representación material y simbólica de la riqueza.

En una perspectiva general y en el contexto de sociedades democráticas y economías de mercado, existen diversos escenarios teóricos para aproximarse a un ejercicio analítico sobre las relaciones riqueza/ vivienda y sobre las funciones del Estado y sus interacciones con el mercado. Unos se refieren a la necesidad de la intervención del Estado para controlar las crisis de la acumulación en los ciclos económicos y garantizar el bienestar general (Keynes y los autores denominados poskeynesianos); otros argumentan un mayor protagonismo del mercado, reconociendo en él un mecanismo óptimo y neutral de asignación de

* Economista, Ph. D. en Ciencias Económicas, Universidad Nacional de Colombia. Docente investigador Maestría en Ciencias Económicas Universidad Santo Tomás.

** Economista, Ph. D. en Economía, Universidad Nacional Autónoma de México. 
recursos, a cambio de un rol discrecional del Estado en el campo de la regulación, las acciones subsidiarias y la compensación en situaciones de fallas de mercado (Friedman y los seguidores del monetarismo neoclásico - escuela de Chicago).

La corriente de pensamiento económico basada en Keynes promueve la perspectiva de los Estados benefactores. Su influencia se observó en las economías occidentales, de manera especial durante las cuatro décadas posteriores a la Segunda Guerra Mundial. A este enfoque se pueden asociar las políticas de vivienda que tuvieron lugar en América Latina, en las cuales el rol del Estado atendía en la práctica todos los encadenamientos de la producción de vivienda, desde la gestión del suelo, pasando por los subsidios y los créditos, hasta el bien final que recibían los beneficiarios de la política.

En cambio, las posturas teóricas basadas en los paradigmas de la escuela de Chicago, sustentaron las transformaciones de los modelos de desarrollo en el mundo occidental desde finales de los ochenta, las cuales llevaron a desmontar en gran medida las estructuras del Estado benefactor y se instauraron instancias institucionales que dieron lugar a un papel estatal más proclive a la regulación. En ese nuevo escenario, de mayor juego de los mercados y limitaciones a la intervención del Estado, cambiaron las políticas de vivienda, con el criterio de la focalización del gasto público a una demanda seleccionada y la participación de diversos actores, públicos y privados, en los encadenamientos de la producción de vivienda.

En un panorama amplio, las dos perspectivas teóricas mencionadas remiten de una u otra forma a las indagaciones sobre las relaciones entre el Estado y el mercado, entre el mercado y los individuos y colectivos sociales, y entre estos y el Estado. De manera particular, para el caso que nos ocupa -la riqueza y la vivienda-, en el espectro de esas relaciones interesa establecer el papel de la fiscalidad, la provisión de vivienda y la regulación del mercado en el sector de la construcción. En el fondo, la relación que pueda establecerse entre la riqueza y la vivienda es similar a la relación que se establece entre la política económica y la política social, mediada por la participación del Estado, cuyo objetivo en el "deber ser" se expresaría en un equilibrio entre la 
eficiencia económica y la conveniencia por el bienestar (Stiglitz, 1977), equilibrio en el que las nociones de equidad, igualdad y justicia, entre otras, hacen parte de las discusiones de la economía pública.

Este texto contiene una aproximación a elementos que permitan una base teórica para desarrollar la investigación sobre riqueza y vivienda en perspectiva de la economía pública. La primera sección se refiere a una distinción de las nociones ingreso, capital y riqueza, con base en aportes germinales de autores reconocidos en la teoría económica; la segunda, incluye reflexiones recientes sobre el hábitat -que dan una noción más amplia a la vivienda, considerada en conjunto como casa y atributos del entorno-, economía y política pública; la tercera, trata sobre vivienda y gasto social; y la cuarta y última sección, presenta el esquema general de la política pública de vivienda en Colombia.

\section{Sobre las nociones de ingreso, capital y riqueza}

Las nociones de ingreso, capital y riqueza, pueden rastrearse en Smith (1776/1997). La noción de ingreso aparece en la frase introductoria del capítulo 1 de su libro Investigación sobre la naturaleza y causas de la riqueza de las naciones. Smith hace una distinción entre la noción que habían acuñado teóricos anteriores a él sobre la riqueza de una nación como un fondo acumulado, con la noción de ingreso -aunque no se haya expresado con ese término-, referido al “... trabajo anual de cada nación que anualmente consume un país”. Es lo que en la terminología moderna de las cuentas nacionales se denomina el producto interno bruto, que no es otra cosa que el flujo anual de los ingresos de un país. En los casos individuales, en la mayor parte de los trabajadores, Smith señala que “... el ingreso deriva exclusivamente de su trabajo" (p. 253), el cual los trabajadores lo agotan en poco tiempo. Hicks (1939/1977, pp. 204-205), añade al trabajo como fuente de ingreso la renta que una persona puede obtener de una propiedad y explica que el ingreso es el valor máximo que puede consumir el individuo durante un tiempo determinado y encontrarse al final de ese período en una situación igual de buena como la que tenía al comienzo del mismo. 
Para la definición de capital, Smith (1776/1997, p. 253) ilustra el caso de la persona que tiene suficientes recursos para mantenerse durante meses o años, reservando una parte necesaria para el consumo y la otra para obtener algún ingreso. A esta última la denomina el capital. Toda la literatura relevante sobre capital circulante y capital fijo se deriva de esa obra germinal y tanto Ricardo como Marx profundizan sobre esas categorías. Ricardo (1817/1987, p. 209) precisa en los siguientes términos la definición dada por Smith: “... el capital es aquella parte de la riqueza de un país que se emplea con vistas a una producción futura, y puede ser aumentado de la misma manera que la riqueza [...], la riqueza depende siempre de la cantidad de bienes producidos".

Marshall (1890/1948, p. 70), señala que “... existe una clara tradición que motiva que se hable del capital cuando se consideran las cosas como agentes de la producción, y que se hable de riqueza cuando se las considera como resultado de la misma, como objetos de consumo y como productora de los placeres que otorga la posesión”. Esta última afirmación hace eco a la definición dada por Smith (1776/1997, p. 31): "Todo hombre es rico o pobre, según el grado en que pueda gozar de las cosas necesarias, convenientes y gratas de la vida".

\section{Hábitat, economía y política pública}

El concepto de hábitat puede entenderse como el espacio geográfico diseñado por los sujetos sociales, un espacio que a su vez es apropiado en diversas formas, magnitudes e intensidades, desde las referidas a las actividades productivas hasta las que referencian imaginarios culturales y simbólicos. Estos últimos trascienden la ocupación física de un territorio y expresan las dimensiones política, socioeconómica, estético-cultural y medioambiental de una sociedad.

La definición del hábitat es un refinamiento derivado de la Declaración Universal de los Derechos Humanos en 1948, la cual constituye el fundamento teórico que ha orientado la actuación de los gobiernos de los países miembros de las Naciones Unidas frente a los problemas 
a los que se ven enfrentados los territorios y las poblaciones cada vez más urbanizadas. En un período de sesenta y ocho años el mundo occidental ha elaborado una noción más comprensiva y amplia del hábitat: mientras en la primera época el problema del hábitat se refería esencialmente a las necesidades de techo en asocio a las necesidades de la reproducción de la fuerza de trabajo y la efectividad de los derechos relacionados con la salud, en las dos décadas finales del siglo xx el concepto de hábitat involucró las ideas de vivienda digna como derecho, asentamientos humanos sustentables y el derecho a la ciudad (PNUD UN-Hábitat CENAC, 2004).

El derecho a la casa ha estado vinculado con el concepto de necesidades básicas insatisfechas (NBI). En cambio, el derecho a la vivienda y el derecho a la ciudad se asocian a la visión de los derechos económicos, sociales y culturales en la perspectiva del desarrollo humano. Junto al concepto de vivienda se enfatiza el aspecto de la tenencia, que no necesariamente se refiere a la propiedad, sino al derecho de usarla.

El derecho a la ciudad incluye aspectos de amplio alcance individual y colectivo, bajo una noción de lo público: espacio público, desarrollo urbano sustentable y equitativo, convivencia y seguridad ciudadana, asociación y participación ciudadana decisoria, transparencia y derecho a ser informado, trabajo y respaldo económico.

Los aspectos económicos del hábitat se refieren esencialmente a su recuperación y sostenibilidad y un medio de hacerlo es la construcción de vivienda. Currie (1983a, 1983b, 1983c, 1987, 1988a, 1988b, 1990) señaló que la construcción de vivienda es una poderosa herramienta de política macroeconómica: en períodos de depresión desempeña un rol dinamizador y permite no solo mitigar los impactos negativos en el conjunto de la economía, sino también promover su reactivación y crecimiento.

Sandilands (1990, pp. 346-347), que fue una de las voces autorizadas sobre la obra de Currie, subrayó la existencia de evidencias de que la construcción de hábitats urbanos y los programas de vivienda se mueven en forma independiente de la tendencia general de la economía. En efecto, se han observado los impactos que una política de financiamiento del hábitat urbano y de vivienda pueden generar sobre 
el crecimiento global de la economía, y también cómo una política de vivienda puede producir rápidamente variaciones muy grandes en la producción corriente en la industria de la construcción y en la demanda agregada. Así, las políticas de hábitat y de vivienda pueden concebirse no simplemente como una política sectorial importante y socialmente útil, sino también como un instrumento de política macroeconómica que puede tener un efecto trascendental sobre las políticas fiscales, monetarias o cambiarias convencionales.

Currie se refirió al principio de insaciabilidad del deseo -elemento base de la teoría económica para comprender el actuar humano-, señalando que aplicado a la demanda de vivienda, explicaría que una rápida expansión del mercado no pondría a la demanda en riesgo de saturación. En opinión de Currie, la construcción y recuperación de hábitat urbano, incluida la construcción de vivienda, tienen la capacidad de generar empleos directos e indirectos (a través de industrias que proveen de insumos al sector) y de impulsar la demanda real de los productos de toda la economía. Los beneficios macroeconómicos se traducen en beneficios sociales extensivos, derivados de empleos mejor remunerados para los pobres, mejor entorno y vivienda para todos y mayores ingresos con base en los cuales se podían financiar programas sociales de toda índole.

Desde el punto de vista de la política pública, la magnitud y la intensidad de los problemas de hábitat, que son distintos en las ciudades y dentro de ellas, imponen retos diversos a una administración pública: debe contrastar las magnitudes e intensidades de los problemas con la capacidad económica, social, institucional y política de la ciudad, establecer las estrategias de crecimiento sostenido y desarrollo humano y actuar en consecuencia. En la perspectiva del hábitat, y en referencia a su dimensión económica y el papel que juega la administración pública, las siguientes preguntas son pertinentes:

- ¿Qué bienes públicos deben producirse?

- ¿Cómo producirlos?

- ¿Para quienes?

- ¿Cómo se toman las decisiones? 
En su propósito de garantizar la sostenibilidad del hábitat en la ciudad, un análisis desde la economía pública permitirá:

- Identificar y definir las acciones que debe realizar la administración pública, como también la forma de llevarlas a cabo, sus recursos y la forma de asignarlos.

- Comprender y anticiparse a los efectos de las acciones que una administración pública decida desarrollar en el área de su jurisdicción para resolver problemas del hábitat y también a las externalidades negativas que puedan derivarse de su actuación.

- Evaluar los efectos e impactos de su actuación.

La previsión de las consecuencias y su evaluación han de permitir valorar la conveniencia de las acciones del mercado y de la política pública en el cumplimiento de objetivos deseados hacia la sostenibilidad del hábitat (bienestar) en la ciudad.

\section{Sobre la vivienda y el gasto social como compensación del ingreso individual}

La vivienda es un bien privado, pero es objeto de políticas públicas que, guardadas algunas diferencias, se han orientado a privilegiar su consumo en ciertos sectores de la sociedad. La naturaleza de la política pública en materia de subsidio remite a la vieja discusión de la distribución de la riqueza. Marshall (1890/1948, p. 193) señalaba al respecto que "Un pueblo en el cual la riqueza esté bien distribuida [...] llegará a acumular una gran cantidad de propiedad pública...”. Las propiedades públicas las definió Marshall como bienes colectivos o propiedad pública material. Es factible deducir de estas apreciaciones el alcance que se le ha dado a las nociones de recuperación y sostenibilidad del hábitat y también, a la importancia que Currie le dio a la construcción de vivienda como dinamizadora de la economía y medio de mejoramiento de las condiciones de vida de la población.

Ahora bien, ¿a quién se debe destinar el gasto social en vivienda? Las encuestas de ingresos y gastos han permitido caracterizar grupos de 
consumidores con "determinadas necesidades”. Hicks (1939/1977, p. 11), hizo una distinción de lo que en su momento constituyó el debate teórico entre la concepción utilitarista ${ }^{1}$ que interpreta "determinadas necesidades" como una función de utilidad - una determinada intensidad de deseo de un conjunto cualquiera de mercancías-, y la concepción que define "determinadas necesidades" como una escala de preferencias $\mathrm{dada}^{2}$. En las páginas subsiguientes Hicks reflexiona sobre dos conceptos: el ingreso-consumo y el precio-consumo y explica cómo, dependiendo del ingreso o de los precios de mercado de los bienes, las personas eligen una canasta de bienes.

Recordando la definición que hace Hicks del ingreso de una persona como el valor máximo que puede consumir en un período de tiempo determinado y relacionarlo con cierto tipo de bienes como la vivienda, es comprensible la situación en la que se encuentran amplios segmentos de población con bajos niveles de ingresos sin posibilidad real de acceder a ella. Es también en esta situación que se comprende la intervención del Estado a través del gasto público en vivienda. El análisis del efecto que producen el gasto del Gobierno en vivienda en el ingreso de un consumidor para que pueda acceder a ella, puede hacerse a partir del procedimiento analítico propuesto por Hicks (1939/1977, pp. 26-27): en la gráfica 1, en el eje horizontal, $X$ representa el bien vivienda y, en el eje vertical, $Y$ representa el conjunto de los bienes restantes de una canasta de consumo, cuyo contenido expresa el poder adquisitivo de los ingresos de un consumidor.

Hicks hace el símil de esta idea con la de "dinero" de Marshall: el precio de una mercancía es igual a la tasa marginal de sustitución del dinero por esa mercancía ${ }^{3}$. Hicks señala que "mientras se conozcan

1 Marshall, Walras, Jevons, entre otros.

2 Pareto fue el primero en afirmar que para establecer las cantidades de mercancías que comprará un individuo a determinados precios, bastaba conocer su mapa de indiferencia.

3 Marshall expuso el supuesto de considerar constante la utilidad marginal del dinero. Hicks (1939, p. 29) hizo la crítica a este supuesto en el sentido de dejar de lado "el efecto que producen sobre la demanda los cambios en el ingreso real que resultan de las alteraciones de los precios". 
las condiciones en que el dinero puede convertirse en otras mercancías, no hay razón para que no tracemos un sistema determinado de curvas de indiferencia, entre cualquier mercancía $X$ (vivienda) y dinero (es decir, poder adquisitivo general)" y, agrega, que este principio es de aplicación general, bajo el supuesto de que los precios relativos de las mercancías que se agrupan en la categoría "poder adquisitivo" no cambian.

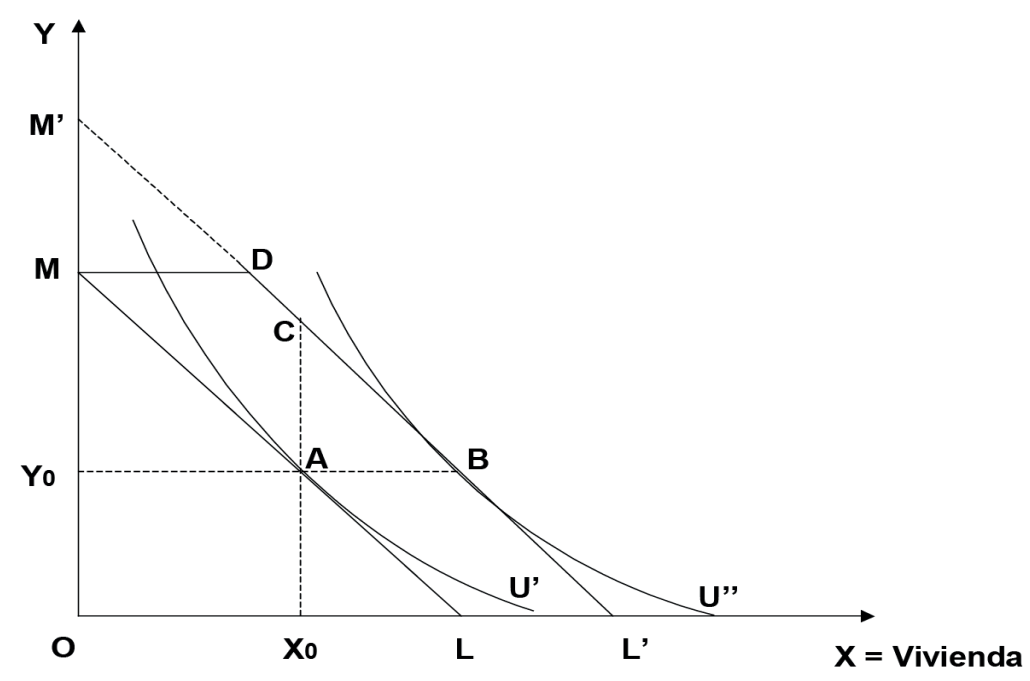

Gráfica 1. Efecto del subsidio de vivienda en el ingreso real

La gráfica 1, permite señalar:

- $\quad$ La línea $M L$ representa el nivel de ingreso de un hogar. El área $O M L$ representa el conjunto de bienes que el hogar está en capacidad de adquirir bajo ese nivel de ingreso (restricción presupuestal).

- El hogar se encuentra en la situación inicial de su nivel de ingreso $M L$, en el punto $A$, donde el presupuesto se encuentra con la curva $U$, referida esta al espacio de la elección de sus preferencias. El supuesto en la situación inicial del hogar en el punto $A$, es la ausencia de propiedad de vivienda, pero parte de sus ingresos lo destina al pago de un alquiler $\left(O X_{0}\right)$. El tramo $\mathrm{OY}_{0}$, equivale a los otros bienes de la canasta de consumo. 
- La única forma de que el hogar no propietario de vivienda con la restricción de su presupuesto en $A$, pueda acceder a una vivienda propia, es a través del aumento de sus ingresos. Ello puede ocurrir mejorando su posición en el mercado laboral o mediante la transferencia de recursos gubernamentales. En cualquiera de los dos casos, el hogar ampliaría su nivel de ingresos representado por un desplazamiento de la recta $M L$ hacia la derecha $\left(M^{\prime} L^{\prime}\right)$.

- En la nueva situación (punto $B$ de tangencia del nuevo nivel de ingresos con la curva de elección de preferencia de bienes y servicios $U^{\prime}$ ), significaría que la asignación de recursos estatales al hogar le permite acceder, según el sistema de precios de mercado, a una vivienda, siempre y cuando en el nuevo nivel de ingresos la proporción de gasto de su presupuesto en vivienda continúe siendo la misma. En otros términos, la política gubernamental no solo estaría permitiendo el acceso a la vivienda, sino también elevando el poder adquisitivo del dinero de ese hogar para ampliar su capacidad de consumo de otros bienes y servicios en su canasta, con lo cual, en términos reales, su punto de tangencia entre su nivel de ingreso y su capacidad de elegir bienes y servicios antes restringidos, no sería $B$, sino $C$ o quizás el punto $E$, tal como se ilustra en la gráfica 2 .

En la caracterización de los hogares por niveles de ingresos, el peso relativo que tiene el gasto en vivienda (ya sea por arrendamiento o por pago de amortización de créditos hipotecarios) frente al gasto en otros grupos de bienes es mayor en los hogares con bajos niveles de ingreso que en los hogares con altos niveles. Tales estudios han permitido a los diseñadores de políticas de subsidios señalar, por ejemplo para el caso colombiano, que los hogares con menos de 2 salarios mínimos al mes ${ }^{4}$-que en teoría serían objeto de subsidio gubernamental para

4 En Colombia, el salario mínimo mensual en pesos no supera los \$700 mil. El costo de una canasta normativa de bienes y servicios para un hogar promedio de 3 a 5 personas es una proporción superior a los 2 salarios mínimos al mes. 


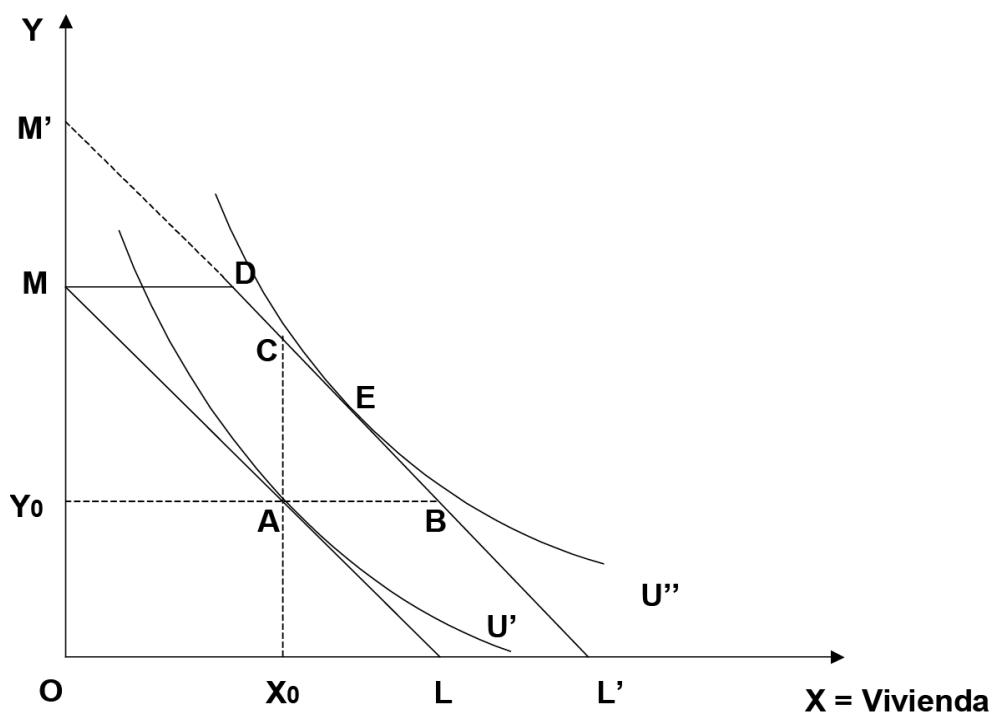

Gráfica 2. Efecto del subsidio de vivienda en el ingreso real y capacidad de los hogares para elegir bienes y servicios

acceder a la vivienda-, no deberían sobrepasar el $30 \%$ del gasto en vivienda sobre el total de los ingresos. En los casos en que sobrepasan este porcentaje, lleva a la argumentación de que los hogares pobres están poniendo en riesgo crítico el consumo de otros bienes de la canasta, entre ellos la alimentación.

Una de las características socioeconómica de quienes devengan menos de 2 salarios mínimos es su condición de trabajador independiente o de trabajador en condición de informalidad. Por lo general, quienes se encuentra en esta categoría y en este rango de ingreso, han demostrado imposibilidad de cumplir con las dos condiciones a las que va atada la decisión gubernamental de otorgar un subsidio: su capacidad de ahorro y su capacidad de endeudamiento para el crédito hipotecario. En muchos casos, si bien logran cierta disciplina de ahorro que les permite alcanzar el porcentaje exigido para el valor de una vivienda normativa, no logran acceder al crédito en el sistema financiero, en razón a su condición de independiente o informal en el mercado laboral.

En todo caso, y al margen de que existan segmentos de la población pobre que no logran realizar el cierre financiero, el objetivo de 
un subsidio de vivienda es complementar el poder adquisitivo de los hogares con bajos salarios, de modo que puedan acceder a la vivienda a través del mercado. El efecto compensación de Hicks es pertinente.

\section{Contexto general de la política pública de vivienda en Colombia ${ }^{5}$}

Existen en Colombia tres leyes que dan marco general a la actuación gubernamental en materia de vivienda dirigida a segmentos de bajos ingresos de la población: la Ley $9^{a}$ de 1989 , la Ley $3^{a}$ de 1991 y la Ley 388 de 1997. Entre la Ley $9^{a}$ y la Ley $3^{a}$, se registra el acuerdo normativo más importante en Colombia en los últimos 25 años: la Carta Constitucional de 1991. Es en ese contexto de finales de los años ochenta y durante la década de los noventa, en el que se presentan los cambios de rol del Estado para el conjunto de las políticas públicas.

En aquellos años, los países en vías de desarrollo alentaron, ante la crisis del Estado de bienestar -denominado Estado intervencionista-, procesos de ajuste institucional y estructural en la economía: descentralización administrativa, política y fiscal, flexibilidad laboral, sistema privado de pensiones y privatizaciones, entre otras. El Estado, además de disminuir su tamaño, asumió el rol de regulador y dejó en manos del mercado un mayor protagonismo en la provisión de bienes y servicios.

En el modelo de desarrollo anterior, de Estado de bienestar, las políticas de vivienda ataron casi todos los espectros de la cadena de la producción de vivienda, desde la gestión de suelo, la infraestructura de servicios y el urbanismo primario y secundario, hasta la construcción de los inmuebles. Durante más de seis décadas, entidades de orden nacional como el Instituto de Crédito Territorial, el Banco Central Hipotecario, el Inurbe, El Fondo Nacional del Ahorro, la Caja de Vivienda Militar, por mencionar algunas del nivel nacional, y la Caja de Vivienda Popular en el nivel distrital, se ocuparon de la provisión

5 Tomado de: Arcos, O., y Casasfranco, M. V. (2007). Diez años de Metrovivienda. ONU-Hábitat, Alcaldía Mayor de Bogotá, Secretaría Distrital de Hábitat, Metrovivienda. 
directa de bienes y servicios de vivienda para la población de escasos recursos, particularmente, aunque algunas no discriminaban los segmentos de ingresos medios.

Otro tanto ocurría en la cadena del consumo, donde el Estado subsidiaba de manera general parte del costo de la vivienda y concedía créditos de largo plazo a la población beneficiaria, a través de las mismas entidades que se ocupaban de la provisión de suelo y la construcción de vivienda. Con las transformaciones normativas y del modelo de desarrollo económico, el rol del Estado cambió sustancialmente en los encadenamientos de producción y consumo de vivienda. Una escala de valoraciones puede ilustrar la participación del Estado en esos encadenamientos, según el modelo de desarrollo de que se trate (tabla 1).

Tabla 1.Valoración de la participación del Estado en la producción y consumo de vivienda, según modelo de desarrollo

\begin{tabular}{|c|c|c|c|}
\hline \multirow[b]{2}{*}{ Ámbito } & \multirow[b]{2}{*}{ Condiciones } & \multicolumn{2}{|c|}{ Modelo de desarrollo } \\
\hline & & $\begin{array}{c}\text { Predominio del } \\
\text { Estado interventor (1) }\end{array}$ & $\begin{array}{l}\text { Predominio del } \\
\text { mercado }\end{array}$ \\
\hline \multirow{3}{*}{ Producción } & Provisión de suelo & Alta & Media \\
\hline & Obras de urbanismo & Alta & Alta \\
\hline & Construcción de vivienda & Alta & Nula \\
\hline \multirow{3}{*}{ Consumo } & Ahorro de las familias & Baja & Nula \\
\hline & Subsidio & Alta & Alta \\
\hline & Crédito hipotecario & Alta & Nula \\
\hline
\end{tabular}

(1) Estado benefactor

En el Estado de bienestar, la incidencia de la gestión gubernamental en provisión de suelo, desarrollo de obras de urbanismo primario y secundario y construcción de vivienda, fue relativamente alta. En el encadenamiento del consumo, la acción estatal también tuvo una alta incidencia en subsidio y crédito, bajo criterios de universalidad, igualdad y oportunidad.

En el nuevo modelo de desarrollo, donde el mayor protagonismo lo tiene el mercado, el Estado tiene a través de los municipios una 
incidencia media en la provisión de suelo ${ }^{6}$, alta en urbanismo primario -en la lógica de provisión de bienes públicos-, y nula en construcción directa de vivienda. En este último aspecto, el Estado solo promueve el desarrollo de programas de vivienda de interés social y la construcción pasó al dominio de las firmas privadas y de organizaciones cooperativas o asociativas populares de vivienda.

En el modelo de desarrollo de Estado interventor y en el de predominio del mercado, la cadena del consumo muestra que el tema del ahorro es de carácter macroeconómico, relacionado con las variables de empleo y salario, además de la distribución del ingreso. El país se ha caracterizado por tener altos índices de concentración del ingreso, empleo mayoritariamente informal y, en el empleo formal, elevados niveles de subempleo y empleos temporales. Así, el tema del ahorro debe valorarse desde la perspectiva estructural de la economía.

En materia de subsidios, el Estado privilegió en el pasado los subsidios a la oferta, con efectos favorables en los precios de las viviendas. Se traducía en una alta incidencia de la acción estatal en la cadena de consumo, a diferencia del presente donde la orientación de la asignación de los recursos públicos involucra criterios de focalización. Como problema relevante habría que señalar que mientras la política de subsidios de vivienda es nacional y homogénea, las situaciones de la población en cada ciudad y municipio son distintas y exigen políticas diferenciadas que corresponden a realidades heterogéneas.

En el nuevo modelo, la incidencia gubernamental en crédito hipotecario es nula: el Estado está en deuda con los más pobres y no ha resuelto de manera decisiva la implementación de mecanismos alternativos como microfinanciación o la banca de oportunidades, entre otras experiencias.

La tríada ahorro-subsidio-crédito, configura en la actualidad el ciere financiero de los hogares para acceder a una vivienda. La focalización del gasto público orientado al subsidio de vivienda ha significado

6 Algunas ciudades del país han conformado bancos inmobiliarios públicos. Salvo el caso de Bogotá, los objetivos de estos bancos inmobiliarios públicos no apuntan a gestión del suelo ni a programas de vivienda. 
grandes partidas del presupuesto gubernamental, con una valoración media/baja en eficacia y eficiencia: de una parte, no se ejecuta la totalidad de los recursos disponibles y, de otra, no llega a los más pobres, porque la mayor parte de ellos pertenecen al sector informal de la economía, en una escala de remuneración por debajo de dos salarios mínimos. La condición de informal de los más pobres indica que las personas, en su mayor parte, no tienen historial bancario y por tanto no son sujetos de crédito. Tal situación impide la concreción de la política de subsidio de vivienda para la población más pobre.

El Estado actúa con limitaciones en la gestión de suelo urbanizado para vivienda de interés social (ámbito de la producción) y en la creación de condiciones económicas y sociales que garanticen el cierre financiero de los más pobres (ámbito del consumo), para que puedan acceder y hacer efectivo su derecho a una vivienda digna.

Los escenarios prefigurados por un modelo de desarrollo con predominio del mercado plantean a la gestión estatal una situación disyuntiva sin solución de continuidad: de una parte, el contrato social de los colombianos determina, en la perspectiva de un Estado social de derecho, que los ciudadanos "... tienen derecho a una vivienda digna"7; de otra, la vivienda es un bien privado sujeto a las condiciones de rivalidad y exclusión en el mercado.

El crédito de vivienda aparece como un factor externo sobre el cual las administraciones locales no tienen el mínimo margen de influencia, porque el sistema de créditos en el país está atado a la política macroeconómica monetaria. Otro tanto puede afirmarse sobre el tema de los subsidios que, en los lineamientos de la política nacional, se hacen efectivos cuando los hogares cumplen con el cierre financiero.

Cualquier análisis objetivo de la gestión de las administraciones locales en materia de política de vivienda tendrá que referenciar

7 "Artículo 51. Todos los colombianos tienen derecho a vivienda digna. El Estado fijará las condiciones necesarias para hacer efectivo este derecho y promoverá planes de vivienda de interés social, sistemas adecuados de financiación a largo plazo y formas asociativas de ejecución de estos programas de vivienda”. (Presidencia de la República, 1991). 
forzosamente la situación del Estado actuando en un modelo de desarrollo con primacía del mercado. En realidad, el Estado solo atiende una parte de los encadenamientos de la producción y del consumo de vivienda.

\section{Conclusiones}

Al menos dos connotaciones se derivan de la conceptualización de riqueza y vivienda desde la perspectiva de la economía pública: la primera se refiere a las actividades inmobiliarias como constitutivas de dinámicas económicas y de acumulación de riqueza y, la segunda, constituye una preocupación permanente de las agendas gubernamentales en materia de política pública. En los dos casos es pertinente una lectura que obligue a las consideraciones macroeconómicas de la acumulación -capital, inversiones, fuerza de trabajo y empleo, tasas de interés y gasto público, por mencionar algunas- y, de igual modo, a consideraciones microeconómicas relacionadas con las posibilidades de acceso a la vivienda por parte de segmentos de bajos ingresos que se constituyen en la población objetivo de las políticas públicas.

La evolución de conceptos que van más allá de la noción de casa e involucran dimensiones del hábitat, lleva a la consideración de formas alternativas de evaluación de la generación y distribución de la riqueza desde un punto de vista sectorial y del papel que desempeñan los gobiernos en esos escenarios. En otras palabras, a la consideración de la eficiencia económica de los mercados inmobiliarios y la conveniencia por el bienestar desde la perspectiva de las políticas públicas.

\section{Referencias}

Arcos, O., y Casasfranco, V. (2007). Diez años de Metrovivienda. ONU-Hábitar, Bogotá D. C., Colombia: Secretaría Distrital del Hábitat, Metrovivienda. Currie, L. (1983a). Nuevas reflexiones sobre oferta y demanda de vivienda. Controversia sobre el Plan de Vivienda sin Cuota Inicial. Camacol, Bogotá D. C., Colombia. 
Currie, L. (1983b). Housing as an instrument of macroeconomic policy. Hábitat International $N^{\circ} 7$, pp. 165-171. En español: Vivienda como instrumento de política macroeconómica, CEDE, 1983, Bogotá D. E., Colombia.

Currie, L. (1983c). La política urbana en un marco macroeconómico. Bogotá D. E., Colombia: Banco Central Hipotecario.

Currie, L. (1987). The Mobilization of Savings for Housing. Policopiado. Informe para UN Hábitat, noviembre, Nairobi, Kenia.

Currie, L. (1988a). Housing Expenditures: Is there a Multiplier Effect? Policopiado. Informe para UN Hábitat, enero, Nairobi, Kenia.

Currie, L. (1988b). The National Accounts of Colombia and Housing. Policopiado. Informe para UN Hábitat, marzo, Nairobi, Kenia.

Currie, L. (1990). Teoría macroeconómica y políticas de crecimiento. Centro de Estudios sobre Desarrollo Económico -CEDE, Universidad de los Andes, Bogotá D. C., Colombia.

Hicks, J. R. (1939). Value and Capital. An Inquiry into Some Fundamental Principles of Economic Theory. Oxford, UK: The Clarendon Press. En español: Valor y capital. México D. F., México: Fondo de Cultura Económica, 1977.

Marshall, A. (1890). Principles of Economics. London, UK: McMillan. En español: Principios de Economía. Madrid, España: Aguilar, 1957.

PNUD UN-Hábitat, CENAC. (2004). Hábitat y Desarrollo Humano. Investigaciones sobre Desarrollo Humano. Bogotá D. C., Colombia.

Ricardo, D. (1817). The Works and Correspondence of David Ricardo. Vol I. On the Principles of Political Economy and Taxation. London, UK: Cambridge University Press. En español: Principios de economía política y tributación. México D. F., México: Fondo de Cultura Económica, 1997.

Sandilands, R. J. (1990). Vida y politica económica de Lauchlin Currie. Bogotá D. C., Colombia: Fondo Editorial Legis.

Smith, A. (1776). An Inquiry into the Nature and Causes of the Wealth of Nations. En español: Investigación sobre la naturaleza y causas de la riqueza de las naciones, Madrid, España: Alianza, 1994.

Stiglitz, J. (1997). La economía del sector público. Barcelona, España: Antoni Bosch Ed., 2a Edición. 
